\title{
PHOTOPHYSICAL PROCESSES AND THEIR ROLE IN POLYMER PHOTOCHEMISTRY
}

\author{
ROBERT B. FoX \\ Naval Research Laboratory, Washington, DC 20390, USA
}

\begin{abstract}
Elementary photophysical processes, well defined in the field of small molecules, are discussed in a polymer context. Examples of most of these processes have been adduced for specific polymers. Intramolecular energy migration is of particular importance in linear vinyl-ring polymers. The relationships between photophysical processes and photochemical processes such as polymer photodegradation are stressed.
\end{abstract}

\section{INTRODUCTION}

With the first plastic material came the first plastic material degradation problem. For many purposes, it was immediately clear that a major causative agent for many kinds of degradation was light. Complicating the problem is the involvement in degradation of other environmental constituents - water, oxygen, atmospheric 'pollutants', as well as constituents of the plastic material itself, added for some specific reason or present as impurities.

Gathered together, these causes have been pointed to the effect called 'weathering'. And generally, 'weathering' has been approached like weather: to avoid sunburn, one keeps his hat on. Likewise, to avoid light-generated degradation in a plastic, one adds something that absorbs the light in preference to the plastic. Occasionally, it was a disconcerting fact that the absorber did more harm than good, and almost always the absorber seemed to be consumed by some photoreaction as it did its work.

In recent years, it has become apparent that the empirical approach to polymer photostabilization is not necessarily the best one. Coupled with the need for improved photostabilizers has come the utilization of photoreactions of polymers in other contexts. Clearly, a knowledge of the fundamental processes involved in the photochemistry of polymers will lead to a more efficient application of photophenomena in the polymer field. Much of this knowledge is already available from studies of the photochemistry and photophysics of non-polymeric organic compounds ${ }^{1-6}$, and it is generally extendable to organic polymers.

Many processes can be and are involved in the interaction between light and an organic material such as a polymer. The ultimately observed photochemical reaction products, both kind and amount, should in principle be controllable through control of the intervening steps. These may be generally 
grouped as shown in Figure 1. It is convenient to think of such steps as photophysical and photochemical processes, with the dividing line between them set by the first bond break occurring following absorption. The photophysical processes are those taking place without chemical change in the system. It is the purpose of this paper to describe in a polymer context those processes that occur between the event of photon absorption and the event of bond dissociation. Particular emphasis will be placed on the photophysical processes that are expected to be more prominent in long chainlike molecules, such as linear organic high polymers, than in 'small' molecules whose dimensions are of the same order of magnitude in all directions.

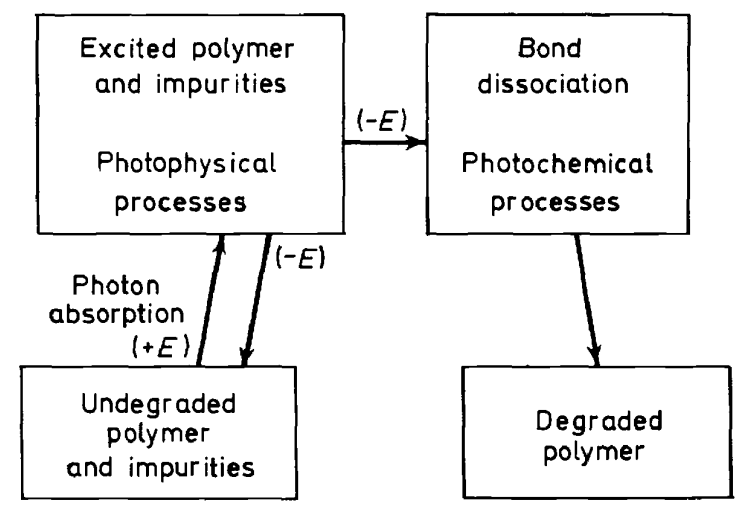

Figure 1. General steps in the overall photochemical process

\section{PHOTOPHYSICAL PROCESSES AND EXCITED STATES IN ORGANIC MOLECULES}

\section{Unimolecular processes}

Most organic molecules contain an even number of electrons. An unexcited molecule in which the electron spins are paired is said to be in the ground electronic singlet state, ${ }^{1} M$, where the term 'singlet' and the superscript refer to the spin multiplicity of the electronic state. Absorption of a photon by the molecule results in excitation of the molecule into a new state of higher energy. If the transition occurs from a ground singlet state without change of spin, the excited electronic state will also be a singlet, ${ }^{1} M^{*}$. Higher excited singlets, ${ }^{1} M^{* *}$ etc., will also be formed if energy of appropriate frequency is absorbed. A plot of the intensity of absorption of the incident light against its frequency or wavelength is called the electronic absorption spectrum. If in an excited state two electron spins become unpaired, a triplet state, ${ }^{3} M^{*}$, will result. Transitions between states of unlike multiplicity are spin-forbidden and occur to a limited extent only because of spin-orbit coupling between the singlet and triplet states. Absorption spectra for the ${ }^{3} M^{*} \leftarrow{ }^{1} M$ transition in principle can also be measured, but the optical densities are usually of the order of $10^{-6}$ of those of the ${ }^{1} M^{*} \leftarrow{ }^{1} M$ transition. 
Absorption of light energy by a molecule to yield an excited state is the first of a series of photophysical processes that may occur before the molecule returns to the ${ }^{1} M$ state or it dissociates; Birks ${ }^{2}$ has discussed the kinetics of these processes in detail. The excited electronic states with their associated manifolds of vibrational levels are shown as a function of energy in Figure 2. The energy of a triplet electronic state is always lower than that of the corresponding singlet state (Hund's rule).

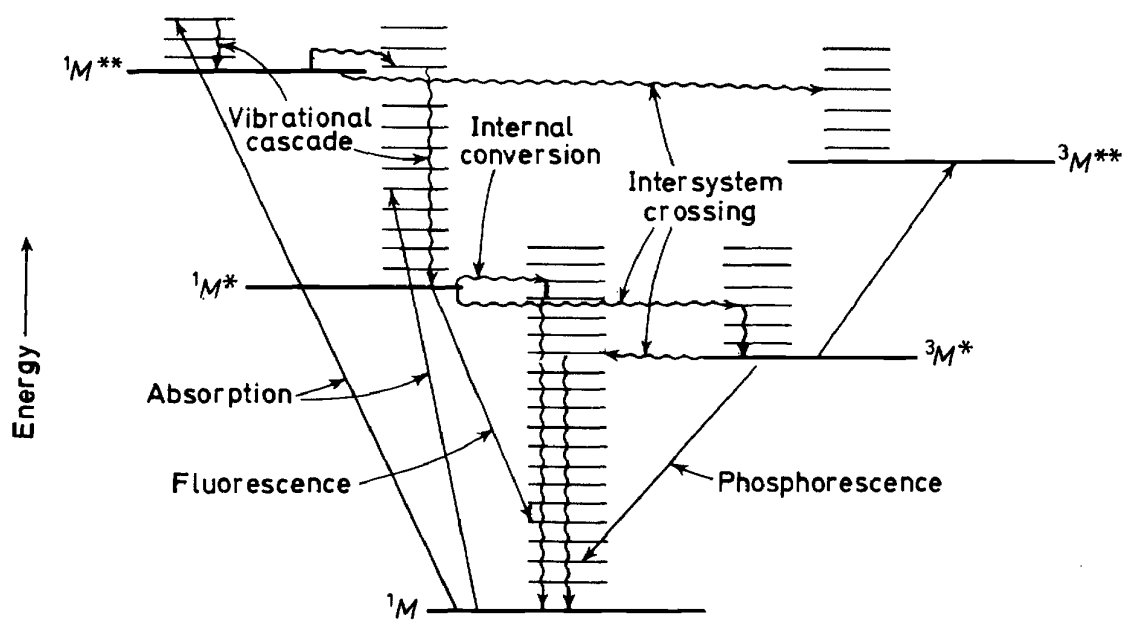

Figure 2. Unimolecular photophysical processes. Solid lines: radiative processes; wavy lines: non-radiative processes

De-excitation of an isolated molecule can take place by radiative or radiationless transitions, shown by solid and wavy lines, respectively, in Figure 2. Fluorescence is radiative emission resulting from a transition between energy levels of like multiplicity; this emission has a short lifetime $\left(10^{-9}\right.$ to $10^{-5}$ s) and usually takes place from the ${ }^{1} M^{*}$ level, although ${ }^{3} M^{* *} \rightarrow{ }^{3} M^{*}$ fluorescence has been observed in a few compounds. Phosphorescence is the radiation from a transition between states of different multiplicity, almost always the ${ }^{3} M^{*} \rightarrow{ }^{1} M$ transition; phosphorescence lifetimes are about $10^{-3}$ to $10^{2} \mathrm{~s}$. The relationships among absorption, fluorescence, excimer fluorescence (see below) and phosphorescence spectra are shown in Figure 3. Fine structure present in the spectra of model compounds is often absent from the spectra of polymers.

Radiationless internal conversion via vibronic coupling from an upper excited level to the lowest excited level $\left({ }^{1} M^{*}\right.$ or $\left.{ }^{3} M^{*}\right)$ is usually a rapid $\left(10^{-12} \mathrm{~s}\right)$ process, and for this reason emission from an upper level is only rarely seen. The same process with a slower rate may take place between the lowest excited state and the ground state, and this competes with the radiative processes of fluorescence and phosphorescence. Intersystem crossing from ${ }^{1} M^{*}$ to ${ }^{3} M^{*}$ is a spin-forbidden radiationless transition that also competes 
with fluorescence; for practical purposes, this is the most important route through which triplets are formed.

The probabilities that these processes will occur in an isolated molecule are dependent on the kind of electronic transition that takes place, i.e. whether the transition is spin, symmetry, and parity allowed, and whether vibronic and spin-orbit coupling are important in the system. In a carbocyclic aromatic ring, as found in polystyrene, the longest wavelength transition is $\pi^{*} \leftarrow \pi$. In an aliphatic ester, such as poly(methyl methacrylate), ketone, acid,

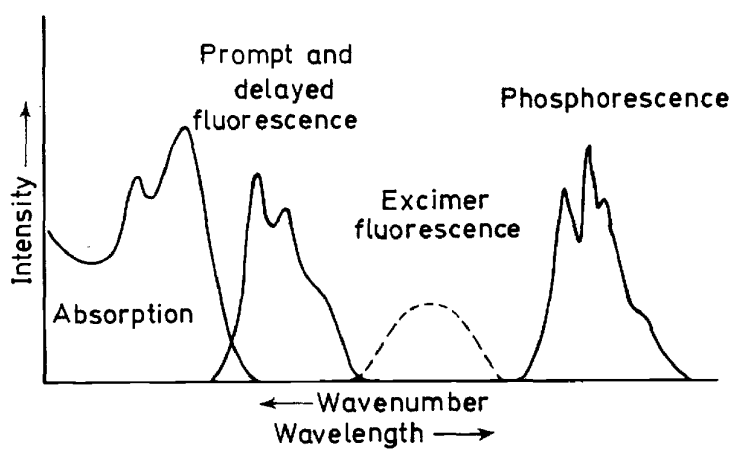

Figure 3. Spectral relationships in absorption and emission bands

or amide, the longest wavelength absorption band will involve a non-bonding electron and the transition will be $\pi^{*} \leftarrow \mathrm{n}$ (symmetry forbidden) with other transitions at much shorter wavelengths. Poly( $N$-vinylcarbazole) and other compounds containing $N$-heterocyclic rings will show both $\pi^{*} \leftarrow \pi$ and $\pi^{*} \leftarrow \mathrm{n}$ as low energy transitions with the energy levels quite close together and sometimes easily transposable by environmental influences or substituents. As a first approximation, spin-orbit coupling is not operative between states of the same configuration ${ }^{7}$. Where a coupling between, say, a singlet $\pi^{*} \leftarrow \mathrm{n}$ and a triplet $\pi^{*} \leftarrow \pi$ state is allowed and the intersystem crossing between them takes place typically in $10^{-9} \mathrm{sec}$, the corresponding transition to a triplet $\pi^{*} \leftarrow \mathrm{n}$ state might require $10^{-5} \mathrm{sec}$. Thus, it would be expected that the ratio of phosphorescence to fluorescence would be greater in poly(1-vinylquinoline) than in poly(1-vinylnaphthalene) because in quinoline there is a $\pi^{*} \leftarrow \mathrm{n}$ triplet lying between the lowest $\pi^{*} \leftarrow \pi$ singlet and $\pi^{*} \leftarrow \pi$ triplet levels, while in naphthalene intersystem crossing must be predominantly between $\pi^{*} \leftarrow \pi$ states $^{8}$.

Other unimolecular processes are less frequently encountered but in certain circumstances they may prove to be quite important. One such is 'E-type' (for eosin) delayed fluorescence, which results from the thermal activation of ${ }^{3} M^{*}$ followed by ${ }^{3} M^{*} \rightarrow{ }^{1} M^{*}$ back intersystem crossing, the reverse of the process shown in Figure 2; the emission will have the spectrum 
of normal fluorescence but a relatively long lifetime (hence 'delayed') depending on the triplet lifetime. This and previously mentioned unimolecular processes are the result of excitation by a single photon.

Under very intense excitation from a light flash or a laser beam, biphotonic processes may also occur. Absorption of a second photon by an excited molecule will raise the energy of the molecule to an upper excited level and could ultimately lead to ionization if the photons are sufficiently energetic. In a typical flash experiment, solutions of polymers and copolymers of 1-vinylnaphthalene are exposed to a 'main' flash to give a triplet population, and this flash is followed almost immediately by a second 'spectral' flash through which the ${ }^{3} M^{* *} \leftarrow{ }^{3} M^{*}$ spectrum and its intensity are measured to determine the extent to which triplets are formed in the polymers under specific conditions ${ }^{9}$.

\section{Bimolecular processes}

While each of the aforementioned processes can take place to a greater or lesser extent in any 'isolated' molecule and certainly can and do take place in organic polymers, it is evident that in practical terms the situation is more complicated. Aside from the problems of chain entanglement even in highly dilute solution, which may lead to special cases of inter-chain interactions, it is clear that intra-chain phenomena may well dominate the photophysical picture due to the close proximity (3-4 $\AA$ ) of adjacent chromophores on a chain. For the present purpose, interactions between chromophores, whether on the same or different chains, can be considered in the class of bimolecular processes.

Perhaps the most ubiquitous of the intermolecular photophysical processes is non-radiative energy transfer, which can involve both singlets and triplets. Transfer of electronic energy occurs during the lifetime of the excited donor molecule, before emission can take place. The act of transfer can be the result of collisional interaction between donor and acceptor, involving a wide variety of mechanisms and excited state intermediates; it may be due to electron exchange interaction over distances of about 6-15 $\AA$; or it can take place by dipole-dipole resonance (Coulombic) interaction over distances between 20 and $60 \AA$, that are large compared to molecular diameters. Experimentally, transfer is readily observed through the quenching of emission from a donor molecule and/or a sensitization of emission from an acceptor having a generally lower energy level than the donor. The phenomenon can be seen in gases, liquids, or solids, although collisional processes are inhibited in rigid systems. In polymers, energy transfer has usually been studied in fluid solution, but it has been observed in a few instances in solid polymers in such systems as poly(1-vinylnaphthalene)-benzophenone ${ }^{10}$.

Energy migration between molecules of the same kind can also take place. Again, the migration may be via either singlets or triplets. It has been studied in greatest detail in organic crystals, but does occur in liquids as well. In crystals, the migration is usually by way of exciton hopping or a coherent exciton wave motion. Since most of the molecules in the crystal lattice are energetically equivalent, absorbed energy is rapidly delocalized throughout the crystal as an exciton wave that migrates until it degrades either radiatively or non-radiatively or is trapped by transfer to a crystal defect or 


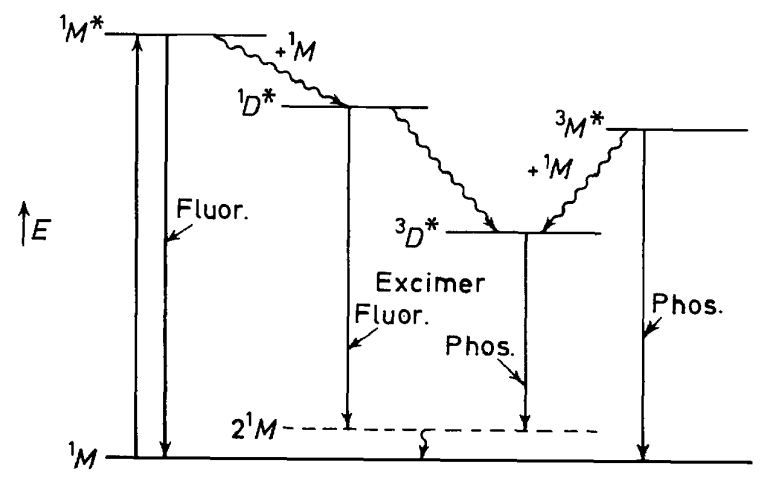

Figure 4. Energy relationships for excimer emission

an impurity. In the latter case, emission from the impurity or defect site is frequently observed. The same kinds of energy migration can also take place in linear polymers, which might be viewed as unidimensional crystals. This will be discussed in detail below.

Of the excited state intermediates formed by bimolecular processes, the best known are the excimers ${ }^{2,11}$, formed by the interaction of an excited singlet molecule with a ground state molecule of the same species. This excited state intermediate is dissociated in the ground state and therefore cannot be detected in the absorption spectrum. Its fluorescence consists of a broad structureless band shifted $4000-6000 \mathrm{~cm}^{-1}$ to the low-energy side of the normal fluorescence (see Figure 3). In a very few cases, excimer phosphorescence has also been observed. Some of the energetic relationships are shown in Figure 4.

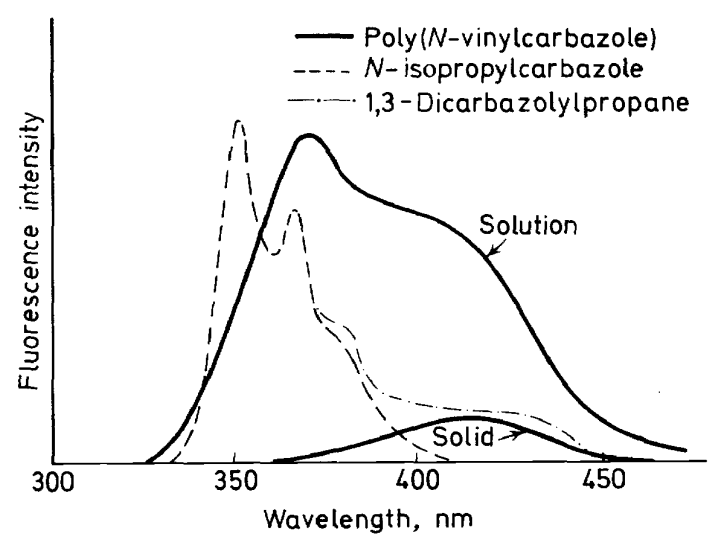

Figure 5. Absorption and emission spectra for poly( $N$-vinylcarbazole) and model compounds 
Intermolecular excimer formation is concentration-dependent, since it is a diffusion-controlled interaction. Intramolecular excimer formation can take place independently of concentration by the interaction of an excited chromophore with another chromophore in the same molecule if the two are in proper geometrical configuration. Hirayama ${ }^{12}$ observed excimer formation in diphenylalkanes only when the phenyl groups were separated by three carbon atoms. Excimer fluorescence is the emission usually observed from room-temperature solutions of vinyl-ring polymers such as polystyrene. An example of an excimer spectrum is shown in Figure 5 for poly $(N$-vinylcarbazole $)^{13}$, together with the spectra for the model compounds 1,3dicarbazolylpropane ${ }^{14}$ and $\mathrm{N}$-isopropylcarbazole ${ }^{15}$.

Excited state intermediates can also be formed between chromophores of different species; in this case, they are termed exciplexes, but their characteristics are similar to those of excimers. Although exciplexes have received less attention than excimers, they may well be more common. An excited state proton transfer interaction in 4-methylumbelliferone, for example, has been made the basis for an organic laser ${ }^{16}$. Exciplexes formed between different aromatic hydrocarbons have been observed, and donor-acceptor excited state complexes, which are a kind of exciplex formed, for example, between aromatic hydrocarbons and tertiary amines, have been studied.

There are many other types of bimolecular photophysical interactions that might be considered ${ }^{2}$. Excited states may be perturbed in the presence of paramagnetic molecules such as oxygen or molecules containing 'heavy' atoms, such as ethyl iodide ${ }^{4}$. The general effect is to enhance spin-orbit coupling in the perturbed molecule and thereby increase the rate of intersystem crossing processes. A particularly important process is that of triplettriplet annihilation, an interaction between two excited states that may lead to excimer formation, the dissociation of which gives an excited singlet state and a ground state molecule :

$$
{ }^{3} M^{*}+{ }^{3} M^{*} \rightarrow\left[{ }^{1} D^{*}\right] \rightarrow{ }^{1} M^{*}+{ }^{1} M
$$

The radiative emission from the ${ }^{1} M^{*}$ is identical to normal fluorescence but with a lifetime proportional to the square of the triplet lifetime. This emission is called P-type (for pyrene) delayed fluorescence, and it has been observed in dilute solutions of certain aromatic polymers such as poly(1-vinylnaphthalene) $)^{17}$.

Each of these excited state intermediates has its own unimolecular photophysical processes. Each, in one form or another, will probably eventually be observed in some polymer system, and each will be as important to the ultimate photochemistry of the polymer as it is in the analogous model compound system.

\section{PHOTOPHYSICAL PROCESSES IN POLYMER MOLECULES}

The development of polymer photochemistry had its genesis in a need to stabilize plastic materials toward weathering. Consequently, much of the research has been of an applied nature and has been oriented toward improvement of material properties. In recent years, however, investigations in polymer photochemistry have been turning toward an understanding of 
photochemical pathways as a means of controlling material properties. Necessarily, this has involved the photophysical processes delineated in the preceding section. In general, these processes have been invoked to rationalize empirical results, but, increasingly, an insight into the processes themselves in polymers is being gained.

Establishment of the nexus between polymer photochemistry and photophysics is receiving emphasis in spite of difficult problems of purification in polymer systems. Pivovarov and co-workers ${ }^{18,19}$ related 'energy transfer' in polystyrene and polypropylene systems to the stabilizing effect of certain additives over and above that resulting from light shielding. In their studies of the photodegradation of ethylene-carbon monoxide copolymers, Heskins and Guillet ${ }^{20}$ implicated the triplet state of the polymer by relating the reduction in quantum yield for chain scission to the concentration of an added triplet quencher, cyclooctadiene. Similarly, the rate of photodegradation of poly(methyl methacrylate) containing an unknown impurity could be roughly correlated with the triplet levels of a variety of inhibitors and accelerators ${ }^{21}$. A necessary background to further research in these directions is information on the most prominent photophysical processes in such systems. It is therefore of interest to examine the detailed emission spectra of highly purified prototype polymers with the object of learning something of energy transfer and migration processes and excited states relative to the phenomena seen in non-polymer molecules.

\section{Intermolecular electronic energy transfer in polymers}

There is abundant evidence pointing to intermolecular electronic energy transfer from polymers to solutes from work in the scintillator field ${ }^{22,23}$. Polystyrene is the most frequently used solvent, and transfer is by way of the polymer excimer (see below) or singlet excited styrene monomer present as an impurity; a wide variety of acceptors such as terphenyl, anthracene, and other common scintillator molecules have been employed. Transfer usually takes place by single-step long-range dipole-dipole interaction.

Lashkov and Ermolaev ${ }^{24}$ investigated solid films of poly $(N$-vinylphthalimide) containing additives, such as benzil or anthracene, having triplet energy levels lower than that suggested by phosphorescence of the polymer. Both a sensitization of the additive phosphorescence and a quenching of the polymer phosphorescence were observed, clearly demonstrating an intermolecular transfer of triplet energy from polymer to additive; transfer among singlet levels was not observed. It was proposed that the act of transfer was preceded by triplet migration along the polymer chains and that the additive molecules acted as traps in a manner similar to energy trapping in doped organic crystals.

Intermolecular energy transfer may also be a biphotonic process. The naphthalene-sensitized photolysis of poly(dimethylsiloxane) at $77^{\circ} \mathrm{K}$ has been shown ${ }^{25}$ by an electron paramagnetic resonance study of the decay of naphthalene triplets and the formation of radicals from the polymer (no naphthalene-derived radicals were observed) to proceed by way of absorption of a photon by the lowest excited triplet state of naphthalene, followed by energy transfer from the upper triplet to the polymer.

As noted above, David and co-workers ${ }^{10}$ have studied the quenching of 
PHOTOPHYSICAL PROCESSES ROLE IN POLYMER PHOTOCHEMISTRY

excimer emission at $77^{\circ} \mathrm{K}$ from solid poly(1-vinylnaphthalene) by benzophenone and by anthracene; sensitized emission from benzophenone was not observed, but anthracene fluorescence was emitted from the system. In vinylnaphthalene-vinylbenzophenone copolymers, no emission was observed from either donor or acceptor. Application of the Förster theory for longrange dipole-dipole singlet interaction showed good agreement between experiment and theory with a critical transfer radius, $R_{0}$, of about $15 \AA$.

David and co-workers have also investigated triplet energy transfer from poly(vinylbenzophenone) ${ }^{26}$ and from poly(phenyl vinyl ketone $)^{27}$ to naphthalene as acceptor in solid films at $77^{\circ} \mathrm{K}$. In both of these systems, triplet formation is a highly efficient process in the polymers as well as in the corresponding model compounds. The systems were excited with $366 \mathrm{~nm}$ radiation, which is absorbed by the polymer but not by naphthalene. Triplet energy transfer took place by the mechanism

$$
{ }^{3} M^{*}+{ }^{1} Y \rightarrow{ }^{1} M+{ }^{3} Y^{*}
$$

where $Y$ is the acceptor naphthalene, the sensitized phosphorescence of which was followed as a function of concentration. For poly(vinylbenzophenone) and poly(phenyl vinyl ketone), $R_{0}$ was $36 \AA$ and $26 \AA$, respectively, which may be compared to the value of $13 \AA$ reported $^{28}$ for the benzophenone-naphthalene system. The difference between the polymers and the model system was attributed to energy migration in the polymer prior to transfer. The nature of the migrating exciton and the question of interversus intra-molecular migration were not considered. Naphthalene was observed to be an inhibitor of the photodegradation of both polymers at room temperature, but it should be noted that degradation mechanisms at room temperature and at $77^{\circ} \mathrm{K}$ need not be identical. Lukáč and co-workers ${ }^{29}$, investigating the inhibition of the main-chain scission photolysis of poly(phenyl vinyl ketone) by triplet quenchers in benzene solution at $30^{\circ} \mathrm{C}$, found that naphthalene as part of the polymer chain in phenyl vinyl ketonevinylnaphthalene copolymers was 21 times more efficient than free naphthalene in quenching the photolysis. This would appear to suggest the idea of some form of intramolecular migration and transfer under these conditions.

Many additional examples of intermolecular electronic energy transfer involving polymers have been reported. In our own laboratory, for example, we have observed in dilute glasses at $77^{\circ} \mathrm{K}$ the quenching of both poly(1vinylnaphthalene $)^{17}$ and polystyrene ${ }^{30}$ phosphorescence by triplet quenchers such as piperylene, and we have sensitized the phosphorescence of poly(1vinylnaphthalene) with benzophenone ${ }^{17}$ by processes analogous to those cited for the poly(vinylbenzophenone)-naphthalene system ${ }^{26}$.

\section{Intramolecular electronic energy transfer in polymers}

Linear organic polymers such as polystyrene differ from their low molecular weight analogues in at least two significant respects: adjacent chromophores on a polymer chain are typically $3-4 \AA$ apart, and polymer chains may have long sequences of such closely spaced chromophores, but the sequences may have internal orientations. Thus orbital overlap between chromophores is easily visualized, and it is a simple step to the concept of some form of energy 
migration through chains of interacting chromophores. Such migration would be crucial in the photochemical fate of a polymer chain, for absorbed energy would seek out an 'energy trap'. If the trap itself dissociated, a main chain break might take place; if the trapped energy were dissipated harmlessly, a stabilization of the chain would result. The latter appears to apply to the phenyl vinyl ketone-vinylnaphthalene copolymers investigated by Lukáč and co-workers ${ }^{29}$.

Delayed fluorescence has been utilized to demonstrate intramolecular triplet migration at $77^{\circ} \mathrm{K}$ in dilute glasses containing poly(1-vinylnaphthalene $)^{17}$, poly(L-tyrosine) or poly(adenylic acid $)^{31}$. Where the polymer emits

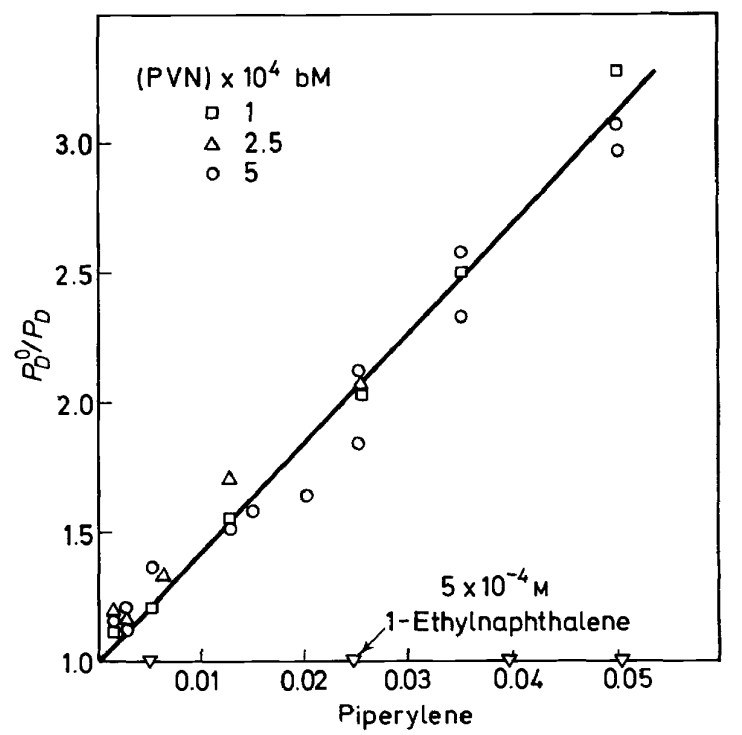

Figure 6. Quenching of phosphorescence of poly(1-vinylnaphthalene) and 1-ethylnaphthalene by piperylene

delayed fluorescence, the low molecular weight model compound [1ethylnaphthalene in the case of poly(1-vinylnaphthalene)] at an equivalent concentration of chromophores shows only phosphorescence in the delayed emission spectrum. The delayed fluorescence was shown to be the result of triplet-triplet annihilation by the finding that the process was second order with respect to the intensity of the exciting light. Intramolecular triplet migration was also supported by the observation that the phosphorescence of the polymer but not that of the model compound was quenched by piperylene ${ }^{17}$. These results are shown in Figure 6.

The intramolecular migration of excitation energy at $77^{\circ} \mathrm{K}$ can be shown in polymers such as polystyrene by incorporating traps as part of the chain, i.e. by utilizing copolymers in which one sequence acts as a trap for energy initially absorbed by a second sequence. Thus, intramolecular migration is 
followed by intramolecular transfer. In Figure 7 is shown an example of such a process in which sequences derived from styrene transfer triplet energy to sequences derived from 1-vinylnaphthalene ${ }^{32}$. Here, the phosphorescence from the styrene sequences is quenched and that from the naphthalenecontaining sequences is sensitized relative to an equivalent mixture of the homopolymers in a dilute glass. The same experiment with monitoring of fluorescence quenching and sensitization shows that singlet energy also migrates and is transferred intramolecularly to the naphthalene sequences in copolymers from styrene and 1 -vinylnaphthalene ${ }^{9}$.

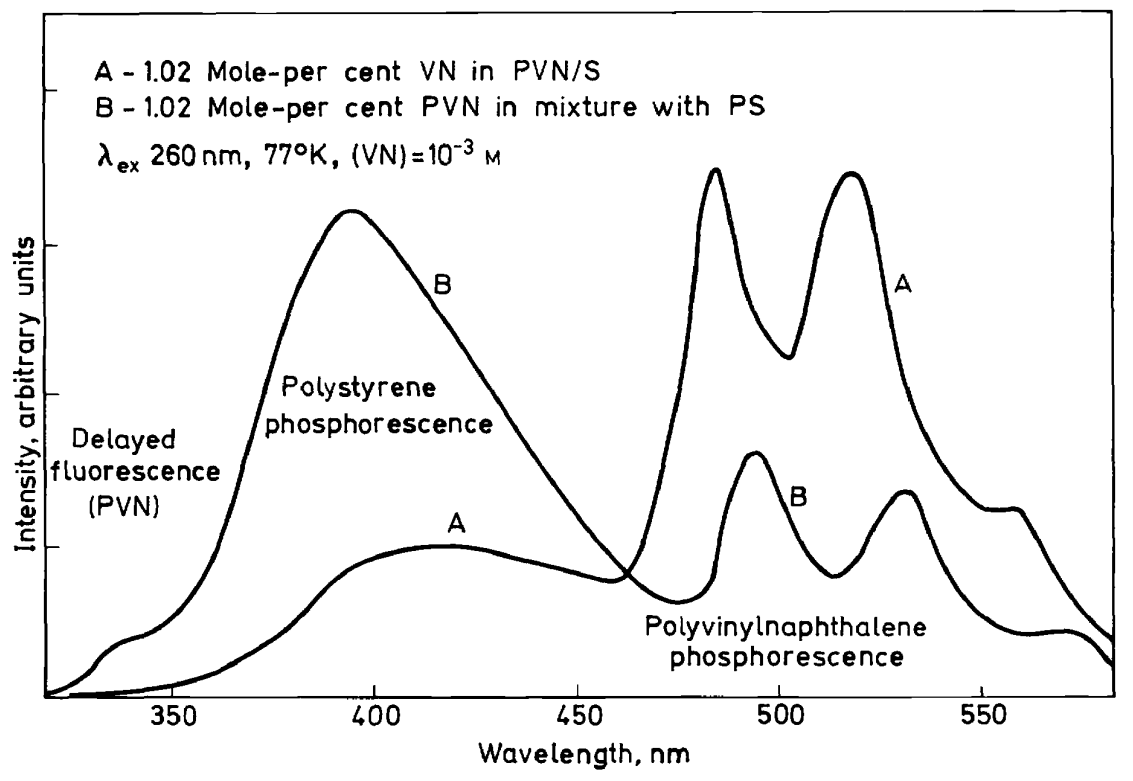

Figure 7. Intramolecular energy migration in styrene sequences and transfer to 1-vinylnaphthalene sequences in a 1-vinylnaphthalene-styrene copolymer

In certain cases where donor and trap segment emission occur at nearly the same wavelength, time-resolved phosphorescence measurements can be used to demonstrate intramolecular triplet migration and transfer. For example, at $77^{\circ} \mathrm{K}$ in a rigid glass, $\tau_{p}$ for polystyrene and poly(4-chlorostyrene) are 3.0 and $0.004 \mathrm{sec}$, respectively. A copolymer of styrene containing two per cent 4-chlorostyrene emits phosphorescence having a $\tau_{p}$ of $0.033 \mathrm{sec}^{9}$.

These kinds of experiments suggest that organic copolymers behave in many ways like unidimensional analogues of mixed organic crystals. Unlike the organic crystals, in which the energy trap may be likened to a point in a tridimensional matrix, the copolymers may contain traps having rather long sequences of chromophores which may themselves be studied. Rise and decay times of the trap emission provide an insight into phenomena within the trap. We have found ${ }^{30}$ that phosphorescence is emitted from sites near 
the ends of the trap sequences and that both $\tau_{p}$ and its energy level are affected by the length of the trap. Delayed fluorescence from within the naphthalenecontaining trap sequences in 1-vinylnaphthalene copolymers is the result of the annihilation of free triplets, while in poly(1-vinylnaphthalene) itself, the annihilation occurs between a free triplet and a triplet localized within the trap, perhaps at some chain defect.

Unanswered questions relate to the nature of the migrating species, the point at which intersystem crossing takes place, and to the absolute rate of migration. Some of these questions are partially answered in the styrene1 -vinylnaphthalene copolymer case by the finding that while piperylene will intermolecularly quench triplets (i.e. phosphorescence) in polystyrene at $77^{\circ} \mathrm{K}$, it does not quench phosphorescence from short-sequence traps in the copolymers ${ }^{30}$. Either energy in polystyrene migrates as singlets (which are not quenched by piperylene) and triplet formation occurs at the point of trapping, or the rate of intramolecular migration of triplets greatly exceeds the rate of intermolecular transfer to piperylene.

The phenomena just discussed concern findings with polymers at quite low temperatures and in rigid dilute solutions. Such migration and transfer processes may very well also take place at room temperatures with fluid solutions or solid polymers, but there is little doubt that there will be other competing processes, not the least of which are the bimolecular interactions. Results such as those obtained with the photodegradation of the phenyl vinyl ketone-vinylnaphthalene copolymers ${ }^{29}$ strongly indicate that some form of migration and transfer does occur in this system in the solid at room temperature.

One photophysical process that stands out in solutions of vinyl-ring polymers at room temperatures is intramolecular excimer formation. Hirayama $^{12,33}$ observed intramolecular excimer emission from fluid solutions of polystyrene and two-unit model compounds for polystyrene, the 1,3-diphenylpropanes. Excimer formation in poly(1-vinylnaphthalene) $)^{34}$, poly $(N \text {-vinylcarbazole })^{13}$, and the corresponding two-unit model compounds have also been reported. In general, intramolecular excimer fluorescence should be observed from polymers derived from compounds that themselves form excimers, provided that certain conformational requirements are met. A fairly specific relationship must exist between adjacent chromophores at the excimer site. Excimer formation in the paracyclophanes ${ }^{34}$ and the diphenylalkanes ${ }^{12}$ indicates that the chromophores should be no more than $3.7 \AA$ apart and should lie in a somewhat parallel conformation. Activation energies for intramolecular excimer formation in 1,3-di(1- and 2-) naphthylpropanes are approximately $4.0 \mathrm{kcal} \mathrm{mol}^{-1}$, a value similar to that for the rotation barrier in a methylene chain ${ }^{35}$.

An example of the variation of fluorescence spectra with temperature where intramolecular excimer formation takes place is shown for poly(2vinylnaphthalene) in Figure $8^{36}$. The solutions are fluid throughout this temperature range, showing that a rigid matrix is not necessary to exclude the formation of excimers. As was done with the 2-unit model compounds ${ }^{35}$, activation energies for intramolecular excimer formation can be determined from plots of the logarithm of the ratio of excimer to molecular fluorescence intensities against $1 / T^{\circ} \mathrm{K}$. Such plots for poly(2-vinylnaphthalene) and 
PHOTOPHYSICAL PROCESSES ROLE IN POLYMER PHOTOCHEMISTRY

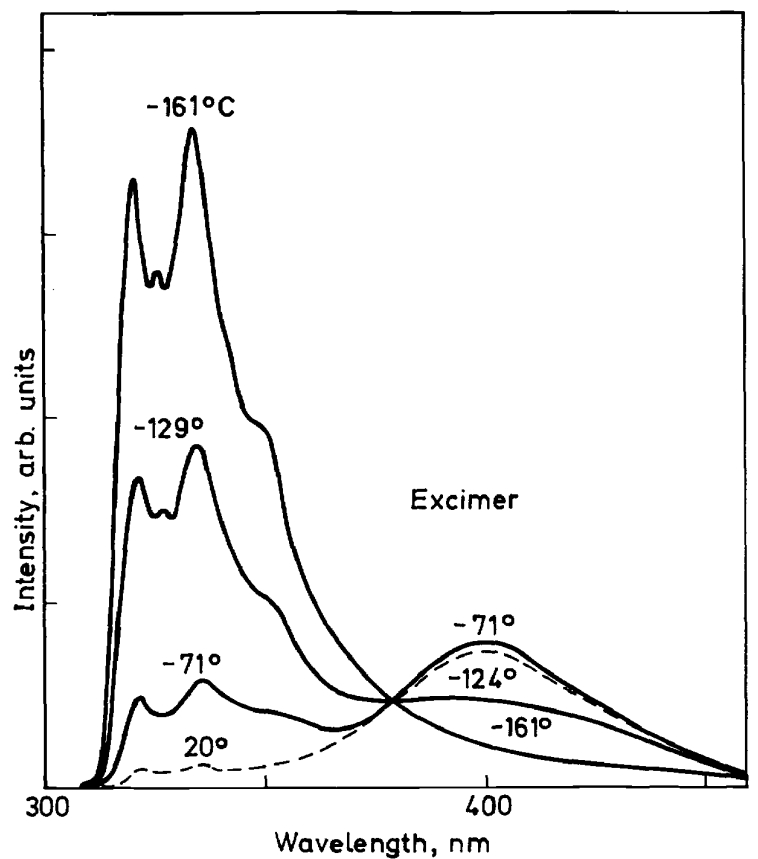

Figure 8. Variation of fluorescence of poly(2-vinylnaphthalene) with temperature

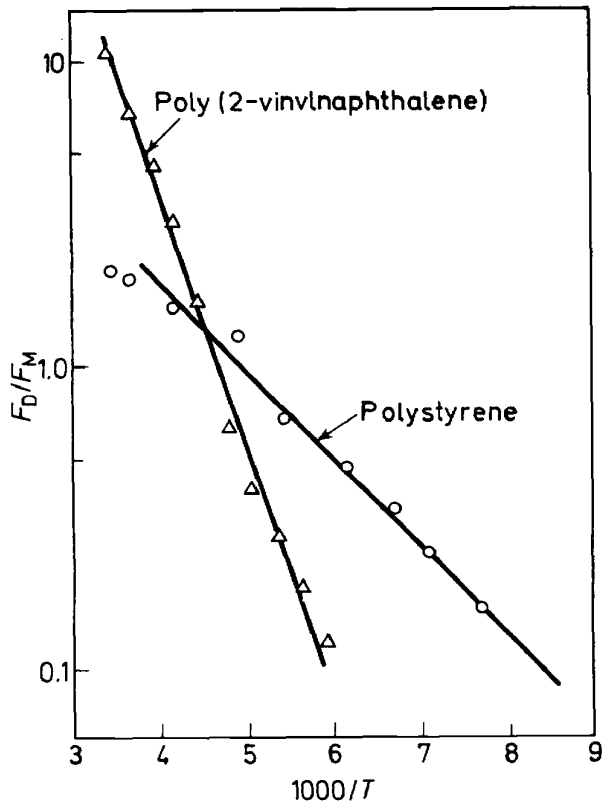

Figure 9. Excimer activation energy plots for poly(2-vinylnaphthalene) and polystyrene 
polystyrene are shown in Figure $9^{36}$. Activation energies of 3.5 and $1.4 \mathrm{kcal}$ $\mathrm{mol}^{-1}$ were obtained. For these polymers, excimer intensities reach a maximum not far below room temperature. The contrast between the 'high' and 'low' temperature regions has been discussed by Birks ${ }^{2}$.

In polymers, a second conformational requirement appears to involve alignment of the chain itself ${ }^{34,37}$. The observation of intramolecular excimer formation in polymers constitutes evidence for intramolecular electronic energy migration. Klöpffer ${ }^{13}$ has shown that in solid poly( $N$-vinylcarbazole) excimer-forming sites and guest molecules compete in trapping migrating

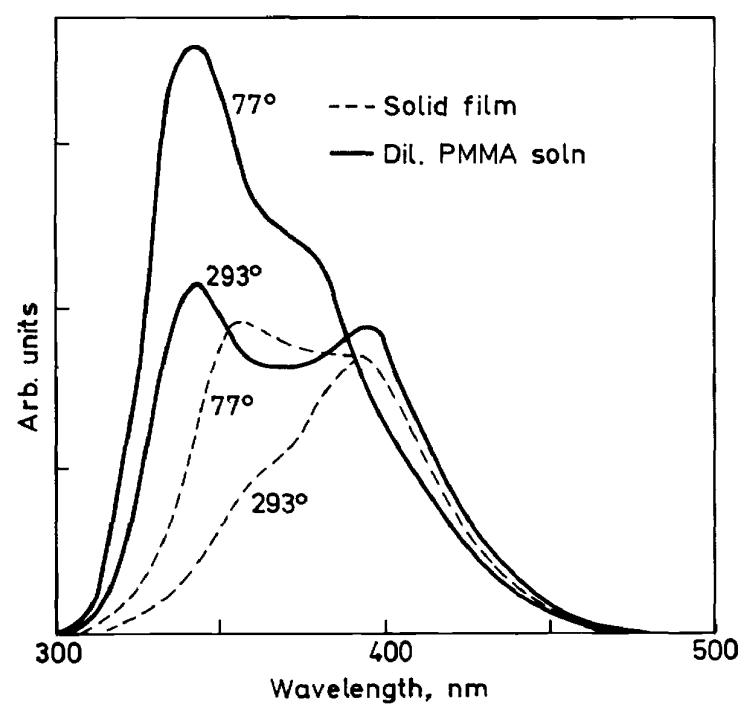

Figure 10. Fluorescence of poly(1-vinylnaphthalene): solid film (solid line); dilute solution in solid poly (methyl methacrylate) (dashed line) (Temperature in ${ }^{\circ} \mathrm{K}$ )

excitons. Energy transfer from an excimer can take place by the dipoledipole mechanism ${ }^{13,33}$. In Figure 10 is shown the fluorescence spectrum of poly(1-vinylnaphthalene) as a solid film and as a solid solution in poly(methyl methacrylate) at room temperature and at $77^{\circ} \mathrm{K}^{36}$. Unlike the fluid solution, the solid shows little decrease in excimer emission as the temperature is lowered. At $77^{\circ} \mathrm{K}$ in a rigid glass formed at low temperatures, excimer formation is absent ${ }^{34}$. It would appear that chain conformation at the time the rigid matrix is formed is the important criterion.

Clearly, excimer formation must influence the photochemistry of these polymers. If the excimer acts as a singlet energy trap, there arises the question of whether this trap is a source of observed photochemical reactions such as degradation. It is possible that some other excited state such as the triplet is the source of bond-breaking. We have found that the triplet population is to 
a large extent controlled by the extent of excimer formation in a series of styrene-1-vinylnaphthalene copolymers ${ }^{9,36}$. As the fraction of 1-vinylnaphthalene-derived units in the copolymer increased, excimer fluorescence intensity also increased. At the same time, the triplet population, measured by the intensity of the ${ }^{3} M^{* *} \leftarrow{ }^{3} M^{*}$ absorption, decreased. It remains to be shown the extent to which each of these excited states is, in fact, responsible for photochemical degradation in vinyl-ring polymers.

\section{SOME APPLICATIONS TO POLYMER PHOTOCHEMISTRY}

Armed with information on the fundamental photophysical processes occurring in any or all constituents in a given polymer system, the polymer chemist not only can do much to explain the empirical observations made during light-induced polymer reactions, but he can also apply this information to predictably control the course of these reactions. One might consider as factors controlling the ultimate fate of a molecule that has absorbed light energy : (a) the thermal excitation in a given energy level; (b) the lifetime of the excited state; and (c) the orbital nature of the excited state. On excitation, normally to some high vibrational level within an excited state manifold, the molecule is said to be 'hot'. While the molecule usually returns to the ground vibrational level of the excited electronic state within picoseconds, during its brief 'hot' period the molecule can undergo dissociation to free radicals. On the other hand, the lifetime of an excited state will control its participation in bimolecular reactions. Thus, one commonly observes short-lived fluorescence at room temperature in fluid solution, but phosphorescence produced by long-lived triplets is only rarely seen under these conditions. The nature of the excited singlet state will certainly control the extent to which intersystem crossing to the triplet level will occur. IJote may be made that the lowest excited singlet level is $\pi^{*} \leftarrow \mathrm{n}$ in aromatic carbonyl compounds, characterized by high intersystem crossing rates and short-lived triplets. One might venture on this basis to predict that an aromatic carbonylcontaining energy trap in a polymer chain would be quite efficient as a stabilizer.

Attention in this report has been directed primarily toward the photophysical processes in polymers; the photochemistry of a polymer also may well be dependent on its interaction with some other excited species. A notable example is the attack of singlet oxygen on polymer chains containing unsaturation. Another is the apparent solvent effects seen in solution photodegradation studies of polymers such as poly(methyl methacrylate).

As illustrations of reports in which photophysical processes have been utilized in the interpretation of polymer phenomena, recent energy transfer studies in the polystyrene-pyrene ${ }^{38}$ and in the nylon-6,6-proflavine ${ }^{39}$. systems may be cited. In both papers, dipole-dipole energy transfer mechanisms were implicated. Briggs and McKellar ${ }^{40}$ have suggested that photostabilization of polypropylene by nickel oxime chelates proceeds by a triplet transfer mechanism to the chelate from carbonyl-containing oxidation sites in the polymer. The work of Lukáč and co-workers ${ }^{29}$ establishing an intramolecular transfer reaction in the photodegradation of poly(phenyl vinyl ketone) may again be mentioned. 
Polymers have been frequently used as photosensitizers and photoreactions in polymers by way of external photosensitization have been studied intensively. For example, 'naphthoylated' polystyrene has recently been used to photosensitize the photoisomerization of cis- and transstilbene $^{41}$. Poly(vinylbenzophenone) and poly(phenyl vinyl ketone) have also been used as photosensitizers. Poly(vinyl cinnamate) is an example of a photosensitive polymer with which external photosensitizers have been used.

It is beyond the scope of this report to dwell exhaustively on all the areas of polymer science in which the underlying processes are photophysical ones. Suffice it to say that wherever a light-induced polymer interaction takes place, one or more photophysical processes will be involved. Once these processes can be identified in a given context, the polymer chemist is in a position to achieve a fair degree of control over the photochemistry of his polymer system.

\section{ACKNOWLEDGEMENT}

The author acknowledges with thanks the many valuable discussions he has had with his colleagues, Prof. Robert F. Cozzens of the George Mason College of Virginia, and Dr J. R. McDonald and Mr T. R. Price of the Naval Research Laboratory.

\section{REFERENCES}

1 J. G. Calvert and J. N. Pitts Jr, Photochemistry, Wiley: New York (1966).

2 J. B. Birks, Photophysics of Aromatic Molecules, Wiley-Interscience: London (1970).

3 C. A. Parker, Photoluminescence of Solutions, Elsevier: Amsterdam (1968).

${ }^{4}$ S. P. McGlynn, T. Azumi and M. Kinoshita, Molecular Spectroscopy of the Triplet State, Prentice-Hall: Englewood Cliffs, N.J. (1969).

5 J. N. Pitts Jr, G. S. Hammond and W. A. Noyes Jr, eds., Advances in Photochemistry, Interscience: New York; a continuing series.

6 H. H. Jaffé and M. Orchin, Theory and Applications of Ultraviolet Spectroscopy, Wiley: New York (1962).

7 S. K. Lower and M. A. El-Sayed, Chem. Rev. 66, 199 (1966).

${ }^{8}$ M. A. El-Sayed, J. Chem. Phys. 38, 2834 (1963).

9 R. B. Fox, T. R. Price and R. F. Cozzens, unpublished results.

10 C. David, W. Demarteau and G. Geuskens, Europ. Polym. J. 6, 1397 (1970).

11 Th. Förster, Angew. Chem., Intl. Ed. 8, 333 (1969).

12 F. Hirayama, J. Chem. Phys. 42, 3163 (1965).

13 W. Klöpffer, J. Chem. Phys. 50, 2337 (1969).

14 a W. Klöpffer, Chem. Phys. Letters, 4, 193 (1969).

${ }^{b}$ W. Klöpffer and W. Liptay, Z. Naturforsch. 25a. 1091 (1970).

15 W. Klöpffer, J. Chem. Phys. 50, 1689 (1969).

16 C. V. Shank, A. Dienes, A. M. Trozzolo and J. A. Myer, Appl. Phys. Letters, 16, 405 (1970).

17 R. F. Cozzens and R. B. Fox, J. Chem. Phys. 50, 1532 (1969).

18 A. P. Pivovarov, Yu. A. Ershov and A. F. Lukovnikov, Plast. Massy, 7 (1966).

19 A. P. Pivovarov and A. F. Lukovnikov, Vysokomol. Soedin. A9, 2727 (1967); Polym. Sci., USSR, 9, 3087 (1967).

20 M. Heskins and J. E. Guillet, Macromolecules, 1, 97 (1968).

21 R. B. Fox and T. R. Price, J. Appl. Polym. Sci. 11, 2373 (1967).

22 R. K. Swank and W. L. Buck, Phys. Rev. 91,927 (1953).

23 J. B. Birks, The Theory and Practice of Scintillation Counting, Pergamon: Oxford (1964).

24 G. I. Lashkov and V. L. Ermolaev, Optics and Spectrosc. 22, 462 (1967).

25 S. Siegel and H. Judeikis, J. Chem. Phys. 43, 343 (1965).

26 C. David, W. Demarteau and G. Geuskens, Europ. Polym. J. 6, 537 (1970). 


\section{PHOTOPHYSICAL PROCESSES ROLE IN POLYMER PHOTOCHEMISTRY}

27 C. David, W. Demarteau and G. Geuskens, Europ. Polym. J. 6, 1405 (1970).

${ }^{28}$ M. Inokuti and F. Hirayama. J. Chem. Phys. 43, 1978 (1965).

29 I. Lukáč, P. Hrdlovič, Z. Maňásek and D. Belluš, J. Polym. Sci. A-1,9, 69 (1971).

${ }^{30}$ R. B. Fox, T. R. Price and R. F. Cozzens, J. Chem. Phys. 54, 79 (1971).

31 J. W. Longworth and M. del C. Battista, Photochem. Photobiol. 11, 207 (1970).

32 R. B. Fox and R. F. Cozzens, Macromolecules, 2, 181 (1969).

${ }^{33}$ F. Hirayama, L. J. Basile and C. Kikuchi, Molec. Crystals, 4, 83 (1968).

34 M. T. Vala Jr, J. Haebig and S. A. Rice, J. Chem. Phys. 43, 886 (1965).

35 E. A. Chandross and C. J. Dempster, J. Amer. Chem. Soc. 92,3586 (1970).

${ }^{36}$ R. B. Fox, T. R. Price, J. R. McDonald and R. F. Cozzens, presented at XXIIIrd Congress of Pure and Applied Chemistry, Boston (1971).

${ }^{37}$ I. H. Hillier and S. A. Rice, Proc. Nat. Acad. Sci. Wash. 53, 973 (1965).

38 J. Wilske and H. Heusinger, J. Polym. Sci. A-1, 7, 995 (1969).

39 H. H. Dearman, F. T. Lang and W. C. Neely, J. Polym. Sci. A-2, 7, 497 (1969).

40 P. J. Briggs and J. F. McKellar, J. Appl. Polym. Sci. 12, 1825 (1968).

41 H. A. Hammond, J. C. Doty, T. M. Laakso and J. L. R. Williams, Macromolecules, 3, 711 (1970). 